\title{
Clinical approach in treatment of resistant hypertension
}

\author{
This article was published in the following Dove Press journal: \\ Integrated Blood Pressure Control \\ 29 July 2009 \\ Number of times this article has been viewed
}

Correspondence: Jennifer Frank University of Wisconsin School of Medicine and Public Health, Department of Family Medicine, 229 S. Morrison Street, Appleton, WI 549I I, USA Tel + I 920-997-8498

$\mathrm{Fax}+$ I 920-832-2797

Email jennifer.frank@fammed.wisc.edu

\begin{abstract}
Resistant hypertension, defined as failure to achieve target blood pressure despite the use of optimal or maximum doses of at least 3 agents, one of which is a diuretic, or requiring 4 or more medications to achieve blood pressure goal, is likely to affect up to $20 \%$ of all patients with hypertension. Apparent resistant hypertension may be caused by medication nonadherence, substances that either interfere with antihypertensive mediations or cause blood pressure elevation, and under- or inappropriate medication treatment. Certain patient characteristics are associated with the presence of resistant hypertension and include chronic kidney disease, diabetes, obesity, and presence of end-organ damage (microalbuminuria, retinopathy, left-ventricular hypertrophy). Secondary causes of resistant hypertension are not uncommon and include obstructive sleep apnea, chronic kidney disease, primary aldosteronism, renal artery stenosis, pheochromocytoma, and Cushing's disease. Initial medication management usually includes adding or increasing the dose of a diuretic, which is effective in lowering the blood pressure of a large number of patients with resistant hypertension. Additional management options include maximizing lifestyle modification, combination therapy of antihypertensive agents depending on individual patient characteristics, adding less-commonly used fourth- or fifth-line antihypertensive agents, and referral to a hypertension specialist.
\end{abstract}

Keywords: resistant hypertension, blood pressure, diuretic

\section{Introduction}

Resistant hypertension ( $\mathrm{RH})$ is defined as blood pressure that is above the patient's goal despite the use of 3 or more antihypertensive agents from different classes (one of which should ideally be a diuretic) at optimal doses. ${ }^{1}$ This definition includes people who require 4 or more medications to achieve blood pressure control. Resistant hypertension is distinguished from uncontrolled hypertension. Uncontrolled hypertension includes both patients with inadequately treated hypertension and those with true resistant hypertension. ${ }^{1,2}$ It is usually more difficult to attain systolic blood pressure goals than diastolic blood pressure goals. ${ }^{1}$

Patients with resistant hypertension are at higher risk for end-organ damage such as left ventricular hypertrophy, atherosclerotic plaques, retinopathy, and microalbuminuria than similar patients who have controlled hypertension ${ }^{3,4}$ making both identification of and treatment of patients with RH important in prevention of cardiovascular morbidity and mortality. Additionally, patients with true resistant hypertension are at increased risk for cardiovascular morbidity and mortality compared with hypertensive patients with controlled hypertension or pseudoresistance. ${ }^{5}$ 


\section{Incidence/prevalence}

The exact prevalence of resistant hypertension is unknown ${ }^{1}$ but has been estimated to be $10 \%$ to $20 \%{ }^{6-8}$ Patient characteristics associated with resistant hypertension include older age, higher baseline systolic blood pressure, obesity, excessive salt consumption, chronic kidney disease, diabetes, left ventricular hypertrophy, black race, female gender, and living in the southeastern United States. ${ }^{1,8}$ Certain genetic phenotypes may also promote the development of resistant hypertension, although this area of study is relatively limited. ${ }^{1}$

\section{Pseudoresistance}

Pseudoresistance is uncontrolled hypertension caused by under treatment or treatment with inappropriate agents, incorrect blood pressure measurement, white coat hypertension, or medication nonadherence. ${ }^{9}$ Differentiating true resistant hypertension from pseudoresistance is a key component of patient evaluation. ${ }^{1,3,10}$ This includes: identification of substances that may contribute to or cause elevated blood pressure, identification of secondary causes, evaluation of medication adherence, and establishing correct blood pressure measurement. Two common causes of incorrect blood pressure measurement are not allowing the patient to sit quietly before obtaining the measurement and using a cuff that is too small. ${ }^{1}$

Since up to $30 \%$ of patients with apparent resistant hypertension may evidence blood pressure control on 24-hour ambulatory blood pressure monitoring (ABPM), using 24-hour ABPM is indicated in establishing the diagnosis of true resistant hypertension. ${ }^{9,11,12}$ White coat hypertension may mimic resistant hypertension, and one study found a $20 \%$ to $30 \%$ prevalence of controlled blood pressure as measured by 24-hour ambulatory blood pressure monitoring among patients with resistant hypertension. ${ }^{11}$ White coat hypertension or pseudoresistance should be considered in patients with apparent resistant hypertension who do not have evidence of end-organ damage or who have symptoms of hypotension. ${ }^{13,14}$ In elderly patients, arterial stiffness may cause pseudoresistance because less compressible stiff arteries cause falsely elevated blood pressure readings. ${ }^{15}$

In distinguishing patients with true resistant hypertension from patients with white coat hypertension, certain patient characteristics show a higher likelihood of being associated with true resistant hypertension. These characteristics include: male gender, systolic blood pressure in the office of $\geq 180 \mathrm{mmHg}$, elevated fasting blood sugar, low serum potassium, and evidence of end-organ disease (microalbuminuria, left ventricular hypertrophy). ${ }^{16}$

\section{Factors associated with resistant hypertension}

Obesity is associated with an increased risk of resistant hypertension and higher blood pressure readings in general. ${ }^{1}$ While the reasons for the increased risk of resistant hypertension in obese patients is not known with certainty, factors hypothesized to play a role include sympathetic activation in the kidney, increased activation of the renin-angiotensin system, increased intrarenal pressures from surrounding adipose tissue, and changes to the renal architecture including glomerular injury. ${ }^{17}$

Diabetes and hypertension are closely associated, although the role of insulin resistance in causing hypertension is not defined. ${ }^{1}$ Patients with both diabetes and hypertension are more likely to have uncontrolled hypertension and typically require 2 or more antihypertensive agents to reach blood pressure goals. ${ }^{1,2}$

Ingestion of salt in the diet is associated with both essential hypertension and resistant hypertension. Older patients, African-American patients, and patients with chronic kidney disease may be particularly susceptible to the blood pressure effects of salt intake. ${ }^{1}$

Excessive alcohol consumption as well as use of illicit drugs can be associated with resistant hypertension. ${ }^{1}$ Cigarette smoking can elevate blood pressure for up to 30 minutes and should be considered a potential cause of an elevated blood pressure reading. ${ }^{18}$

Medications, both over-the-counter and prescription, can cause elevated blood pressure and may play a role in resistant hypertension (see Table 1). Common medications that can cause elevated blood pressure include: nonsteroidal anti-inflammatory medications, aspirin, COX-2 inhibitors, decongestants, stimulant medications used for weight loss, narcolepsy or attention deficit disorder, oral contraceptive pills, cyclosporine, and erythropoieitin. ${ }^{1}$ Herbal supplements that act as stimulants such as ma huang or ephedra also can cause elevated blood pressure.

Secondary causes of resistant hypertension include obstructive sleep apnea, chronic kidney disease, primary aldosteronism, renal artery stenosis, pheochromocytoma, Cushing's disease, coarctation of the aorta, hyper- or hypothyroidism, and intracranial tumor ${ }^{1,3}$ and are present in approximately $10 \%$ to $20 \%$ of patients with resistant hypertension who are adherent with prescribed treatment. ${ }^{19}$

\section{Obstructive sleep apnea}

Obstructive sleep apnea (OSA) is independently associated with resistant hypertension with a reported odds ratio of 5.0 in 
one case control study ${ }^{20}$ and has been reported to be present in the majority of patients with resistant hypertension presenting to a hypertension clinic. ${ }^{21}$ Increased severity of OSA is associated with increased risk for resistant hypertension. ${ }^{1,2}$

In a group of patients with resistant hypertension, those with a higher probability of having OSA had a significantly higher prevalence of primary aldosteronism (PA) compared to those with a lower probability of OSA based on a validated questionnaire (36 vs 19\%). ${ }^{22}$ A study of consecutive patients with resistant hypertension found a relationship between severity of obstructive sleep apnea and aldosterone excess. ${ }^{21}$ This may indicate that obstructive sleep apnea stimulates aldosterone excretion leading to an increased risk of PA and therefore RH in patients affected by OSA. Alternatively, another factor such as obesity may increase risk for both OSA and excess aldosterone excretion. ${ }^{22}$ Hypoxia experienced by patients with OSA likely leads to sympathetic nervous system activation which in turn raises blood pressure. ${ }^{1}$

Treatment of OSA with a continuous positive airway pressure (CPAP) device has been shown to reduce blood pressure levels, with the greatest benefit seen in those with severe OSA who were already receiving antihypertensive treatment.

\section{Primary aldosteronism}

Approximately $10 \%$ to $20 \%$ of patients with resistant hypertension have primary aldosteronism (PA). ${ }^{1,23}$ The prevalence of PA tends to increase with increasingly severe hypertension. ${ }^{24}$ PA may be associated with both obesity and OSA. ${ }^{1}$

Aldosterone has been demonstrated to exert a number of influences leading to increased systemic vascular resistance including an association with endothelial dysfunction, vascular remodeling through collagen deposition, vascular damage, impairment of the baroreflex leading to loss of compensation for elevated blood pressure, and hypervolemia.,24,25 Aldosterone may promote both renal and cardiovascular injury by mechanisms other than blood pressure elevation. ${ }^{24}$ Aldosterone excess, in the absence of diagnosed primary aldosteronism, may contribute to resistant hypertension as evidenced by the benefit of aldosterone antagonists in patients both with and without PA as well as the demonstration of biochemical markers of aldosterone excess in patients with $\mathrm{RH} .{ }^{8}$ Primary aldosteronism may represent the end-point of a continuum that starts with a low-renin, normotensive state and progresses through low-renin hypertension to normokalemic primary aldosteronism and finally hypokalemic primary aldosteronism. ${ }^{26,27}$

Primary aldosteronism may be suggested by hypokalemia, ${ }^{7}$ although this may be a late finding of the disease process, occurring after hypertension has already developed. ${ }^{1}$ PA is equally prevalent among both black and white patients with RH. ${ }^{7}$

Since primary aldosteronism is often effectively treated with an aldosterone antagonist, it remains controversial whether establishing the diagnosis is necessary in those patients who screen positive with an elevated aldosteronerenin ratio. ${ }^{28,29}$ While adrenal adenectomy is indicated when an aldosterone-producing adenoma is found and can be curative of primary aldosteronism and hypertension, most patients found to have primary aldosteronism do not have an identified adenoma and can be medically managed. ${ }^{28}$

\section{Chronic kidney disease}

Multiple factors likely cause hypertension in patients with chronic kidney disease. These include intravascular volume expansion and activation of the renin-angiotensin system. ${ }^{30}$ Other factors hypothesized to play a causative role include activation of the sympathetic nervous system by decreased blood flow to the kidneys or hypoxemia during sleep apnea events, alterations in endothelium derived vasoconstrictors and vasodilators, increased arterial stiffness, reactive oxygen species (possibly secondary to vasoconstriction), renal ischemia, or recombinant human erythropoietin. ${ }^{30}$ Uncontrolled hypertension in patients with chronic kidney disease is common with an elevated serum creatinine being a strong predictor of resistant hypertension. ${ }^{1}$

\section{Renal artery stenosis}

While renal artery atherosclerotic disease is relatively common in hypertensive patients, what role obstructive lesions play in the elevated blood pressure of these patients is not known. ${ }^{1}$ In patients with resistant hypertension, renal artery atherosclerotic disease is a common secondary cause of hypertension, with a higher prevalence among older patients, smokers, patients with atherosclerotic vascular disease, and patients with unexplained renal insufficiency. ${ }^{1}$ Treatment of renal artery stenosis from atherosclerotic disease with surgical or endovascular revascularization has not been reliably shown to improve blood pressure control. ${ }^{1}$ In contrast, angioplasty of fibromuscular disease causing renal artery stenosis (RAS) can be curative and is almost always beneficial. ${ }^{1}$ Renal artery disease is most commonly related to atherosclerotic plaques, fewer than $10 \%$ of lesions being fibromuscular. ${ }^{1}$

\section{Pheochromocytoma}

While rare, pheochromocytoma may be found in a small minority of patients with RH. Elevated blood pressure occurs 


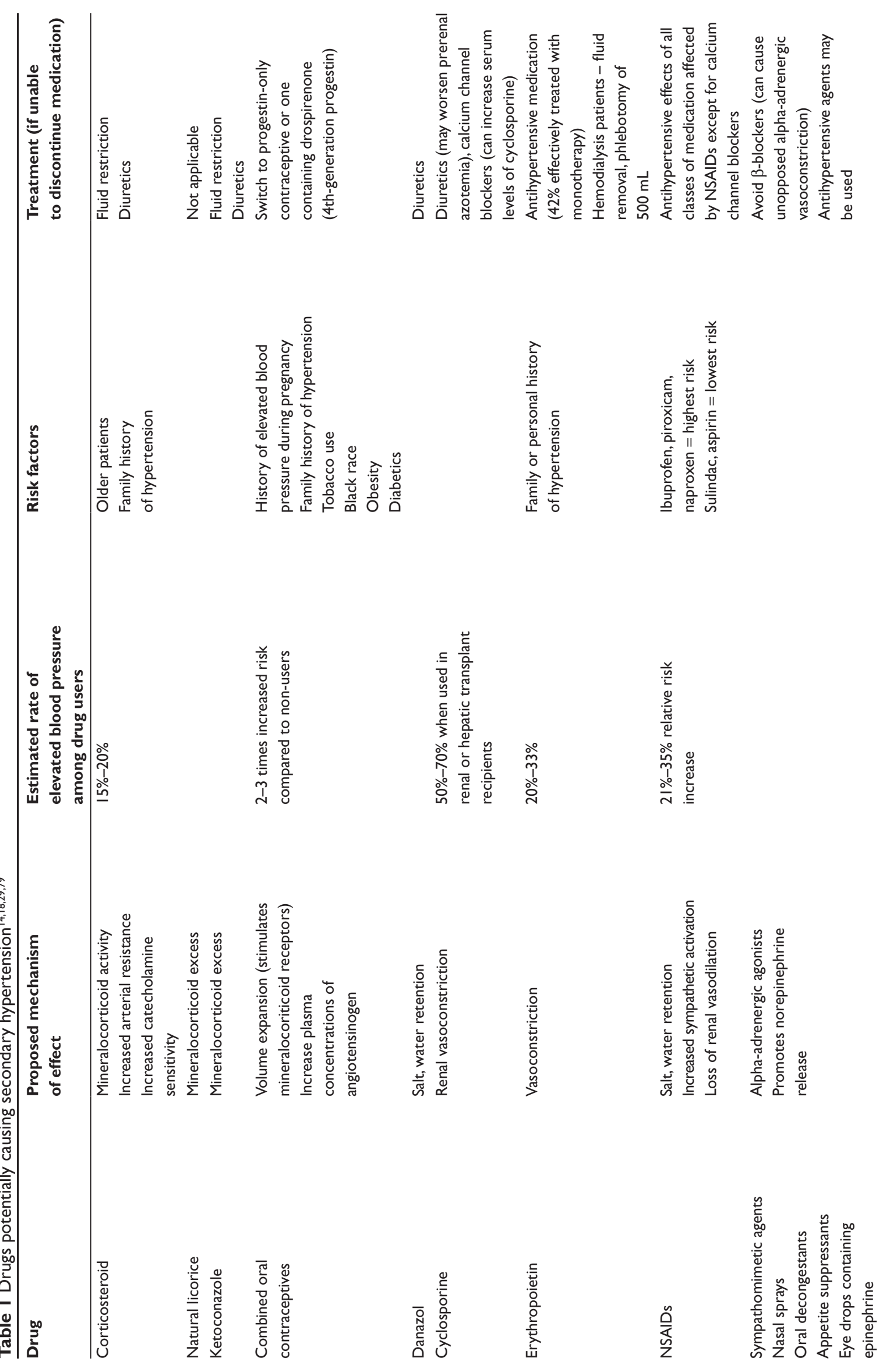




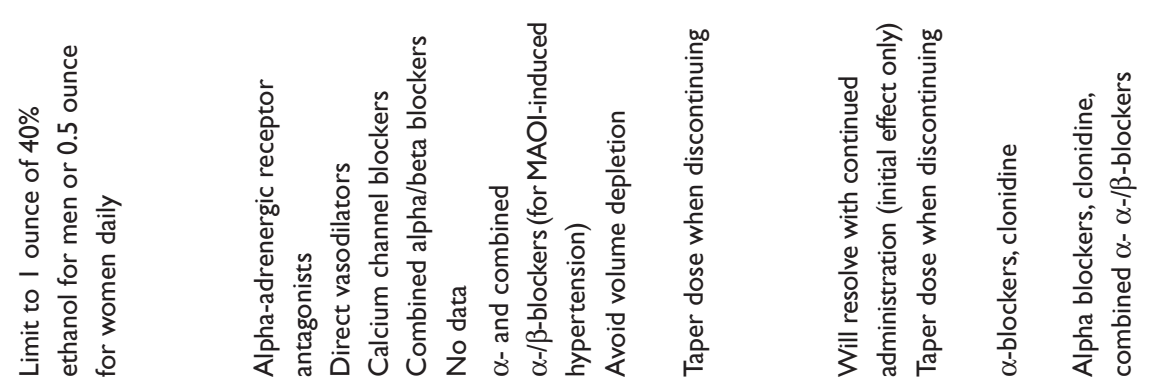
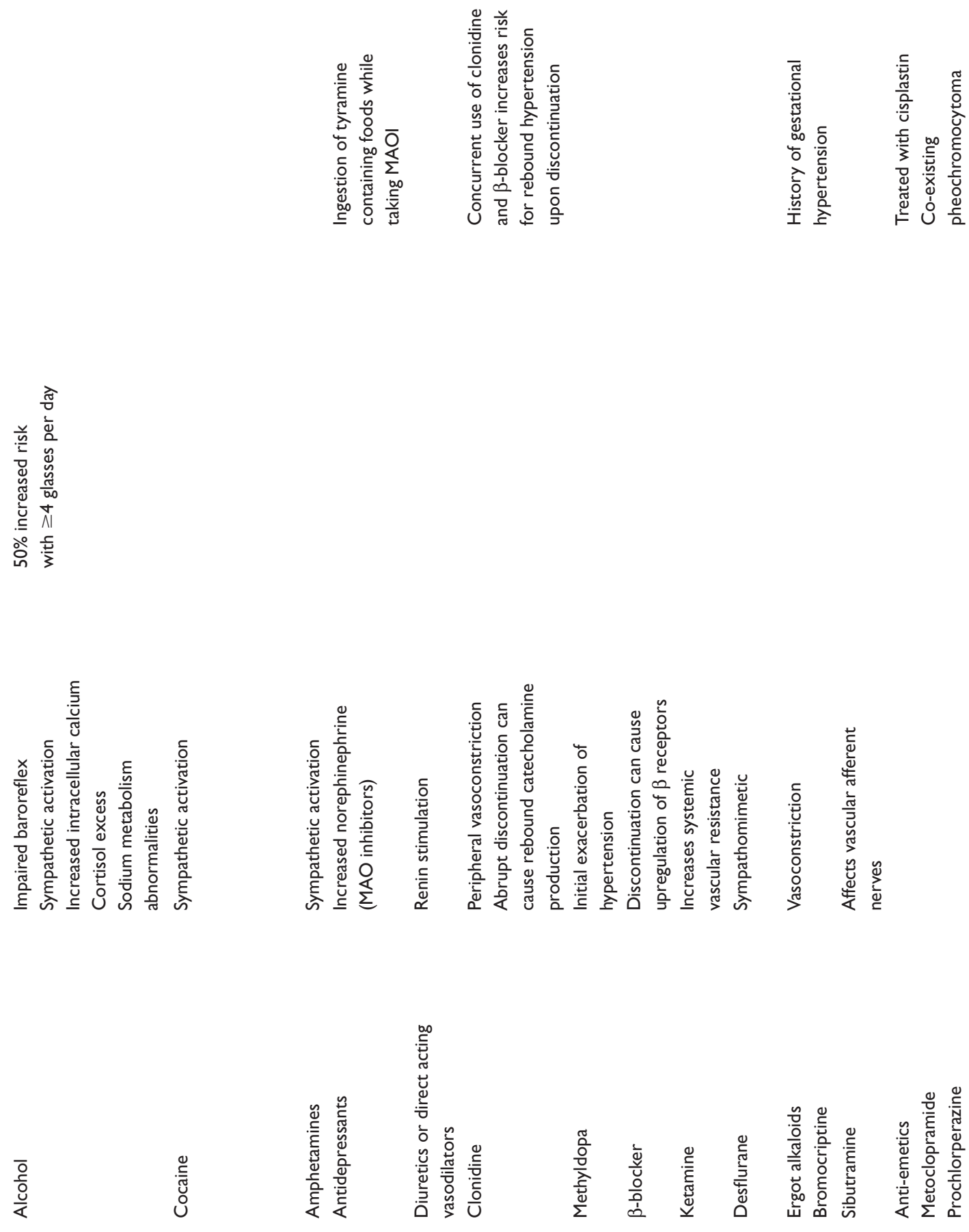
in $95 \%$ of patients with pheochromocytoma, although only about half of patients have persistently elevated blood pressure. ${ }^{1}$ Pheochromocytoma should be considered in a patient with resistant hypertension who experiences headaches, palpitations, and sweating. ${ }^{1}$

\section{Cushing's syndrome}

Elevated blood pressure is common in patients with Cushing's syndrome with a prevalence of resistant hypertension that may be similar to the general population of patients with hypertension (about 1 in 5). ${ }^{1}$ The pathophysiologic mechanism of blood pressure elevation in Cushing's syndrome can result in most antihypertensive agents being ineffective in lowering blood pressure. ${ }^{1}$ Mineralocorticoid receptor antagonists are particularly effective antihypertensive agents in patients with Cushing's syndrome since blood pressure elevation results from cortisol-mediated activation of the mineralocorticoid receptors. ${ }^{1}$ However, other factors can play a role in blood pressure elevation including OSA and insulin resistance. ${ }^{1}$

\section{Patient evaluation}

Resistant hypertension is not uncommonly linked to other medical conditions, and patient evaluation should focus on historical features which may suggest related conditions (see Table 2). Obstructive sleep apnea should be considered in any patient with daytime somnolence, snoring, and witnessed apnea. RAS should be considered in patients with signs or symptoms of peripheral or coronary artery disease. Pheochromocytoma should be considered when labile hypertension is accompanied by palpitations and diaphoresis.

Nonadherence to prescribed antihypertensive medications is common with reported prevalence at $40 \%$ to $60 \%$. $^{1,18}$ Medication nonadherence is more common in chronic, asymptomatic diseases and for treatment that lasts longer than 6 months, making non-adherence in the treatment of hypertension a particular problem. ${ }^{12}$ Therefore, patients should be assessed for medication adherence at each visit and particularly with apparent resistant hypertension. Clinicians should be aware of factors which may increase the risk of patient nonadherence such as medication side effects, cost, and dosing inconvenience of prescribed medications ${ }^{1}$ as well as poor patient understanding of medication purpose and use or the presence of organic brain dysfunction impairing the patient's ability to take medications as prescribed. ${ }^{18}$ Additional information solicited from patients may include whether the patient understands which medications are prescribed for hypertension, how medications are organized, stored, and administered at home, and whether medications are being transferred from old prescription bottles or shared with other family members. Family members are another potential source of information and may be particularly good resources when assessing medication adherence in older or disabled patients who may be more dependent on family assistance in securing and administering medications. ${ }^{1}$ Clinicians can promote medication adherence through patient education, selecting once-daily dosing regimens when possible, avoiding drug interactions, and providing reminders and positive feedback on improved blood pressure values. ${ }^{19}$

Assessing adherence can be challenging but is a necessary component in the evaluation of a patient with apparent resistant hypertension. Use of an electronic monitoring system improves detection of patient's non-adherence with prescribed medications and is superior to pill counting or physician estimates of adherence. ${ }^{31}$ Several issues regarding adherence to prescription medication regimens should be considered. The first is that adherence is a dynamic process - varying over time and improving right before a scheduled office visit. ${ }^{31}$ Additionally, the type of antihypertensive medication plays a role with angiotensin receptor blockers (ARBs) being associated with the highest likelihood of continued adherence. ${ }^{31}$ Finally, electronic monitoring of adherence, beyond improved identification of poor adherence, has been demonstrated to improve blood pressure control. ${ }^{31}$ If questions remain about medication adherence, consideration should be given to a short term hospitalization for closer observation. ${ }^{19}$

\section{Physical examination}

Establishing the diagnosis of resistant hypertension starts by properly evaluating blood pressure measurement and excluding white coat hypertension. ${ }^{1}$ Clinic blood pressure measurement can be falsely elevated due to a number of factors including room temperature, recent patient exercise, nicotine, alcohol or other recent substance use by the patient, incorrect arm position of the patient, talking by the clinician or patient and inability of the clinician to hear the Korotkoff sounds. ${ }^{32}$ Clinic measurement of blood pressure should occur after the patient has been sitting for at least 5 minutes. The patient should be seated with both back and arm supported and legs uncrossed. The blood pressure cuff should not be placed over any clothing and clothing should be removed to avoid a constricting effect on the upper arm. ${ }^{32}$ The patient's arm should be at heart level with a cuff that encircles at least $80 \%$ of the arm circumference. ${ }^{32}$ Clinicians can demonstrate two types of digit bias - both for a terminal zero (ie, 120 instead of 122) and for a reading indicating that goal 
has been achieved. ${ }^{32}$ It is recommended that 2 measurements be taken at least 1 minute apart and averaged to obtain the blood pressure reading. ${ }^{32}$

The physical examination of a patient with suspected resistant hypertension should focus on identification of both micro- and macrovascular complications. ${ }^{1}$ The presence of end-organ damage supports a history of uncontrolled hypertension. ${ }^{1}$ Evaluation for end-organ damage includes a fundoscopic examination looking for retinopathy and a cardiovascular examination looking for bruits and displacement of the PMI indicating left-ventricular hypertrophy. ${ }^{1}$ Additional historical and physical exam findings that may indicate secondary causes of hypertension should be sought (see Table 2).

\section{Laboratory evaluation}

Laboratory evaluation of a patient with known or suspected resistant hypertension includes a hematocrit, basic metabolic panel, a urinalysis including testing for microalbumin, lipids, glucose, uric acid, thyroid stimulating hormone (as thyroid disease is associated with hypertension), and a screen for primary aldosteronism (see below). ${ }^{1,3,12}$ Serum creatinine should not be relied upon to identify chronic kidney disease and a glomerular filtration rate using the Modification of Diet in Renal Disease equation should be calculated in patients with resistant hypertension. ${ }^{12,29}$ If pheochromocytoma is suspected, a urine or plasma test for fractionated free metanephrines (ie, metanephrine and normetanephrine) is indicated. The plasma test has a reported $99 \%$ to $100 \%$ sensitivity and an $89 \%$ to $97.6 \%$ specificity. ${ }^{1,33}$ Testing for microalbuminuria is important for identification of end-organ damage that provides prognostic information on cardiovascular risk but also because it may affect choice of antihypertensive medications. ${ }^{18}$

Screening for primary aldosteronism can be done with plasma renin and serum aldosterone ratio measurement (which has a high sensitivity but low specificity) and confirmed with sodium loading or fludrocortisone suppression testing. ${ }^{12,23}$ While measurement of serum aldosterone - plasma renin ratio has a high negative predictive value, it may be falsely positive up to $50 \%$ of the time. ${ }^{23}$ Confirmatory testing of primary aldosteronism can be done with infusion of $2 \mathrm{~L}$ of normal saline over 4 hours followed by measurement of plasma aldosterone. Failure of the saline infusion to suppress aldosterone to less than 5 to $10 \mathrm{ng} / \mathrm{dL}$ indicates primary aldosteronism. ${ }^{28}$ Alternatively, 24-hour urine excretion of aldosterone can be measured as an outpatient during dietary sodium loading. ${ }^{28}$ Hypokalemia is not a reliable indicator of PA as it may be normal early in the disease course. ${ }^{23,24}$

\section{Diagnostic testing}

Electrocardiography (ECG) is indicated in patients with resistant hypertension to evaluate for left ventricular hypertension (LVH). ${ }^{3,12}$ LVH can both support the diagnosis of resistant hypertension, as end-organ damage is more commonly found in patients with true resistant hypertension, as well as identify patients at increased cardiovascular risk. Echocardiography is more sensitive in detecting LVH but is also more expensive. It may be considered in patients in whom a strong suspicion exists for LVH and may be particularly useful in men older than 50 years for whom the pretest probability is higher. ${ }^{34}$

Renal artery stenosis due to atherosclerosis is common in older patients. Screening for RAS can be done using magnetic resonance angiography (MRA), computer tomographic angiography (CTA), Doppler ultrasonography, or angiotensinconverting enzyme (ACE) inhibitor renography. ${ }^{12,35} \mathrm{MRA}$ or Doppler ultrasonography are preferred in patients with renal impairment. ${ }^{35}$

\section{Treatment}

Treatment of resistant hypertension focuses on several components. These include maximizing therapeutic lifestyle changes, withdrawing any medications or substances which may contribute to elevated blood pressure, treatment of underlying conditions (such as obstructive sleep apnea) (see Table 3), maximizing medication adherence, and focusing on pharmacologic modalities likely to achieve target blood pressure. ${ }^{1}$

Nonpharmacologic treatment focuses on weight loss, dietary salt restriction $(<100 \mathrm{mEq} / 24$ hours $)$, decreased alcohol ingestion, increased physical activity, and ingestion of a high-fiber, low-fat diet rich in fruits and vegetables. ${ }^{1,36}$ Weight loss has not been specifically studied in patients with RH but has been found to reduce both systolic and diastolic blood pressure in hypertensive patients. ${ }^{1,37}$ A low salt diet may be more likely to benefit patients who are at increased likelihood of salt-sensitivity, including older patients, African-American patients, and those with chronic kidney disease. ${ }^{37}$

The timing of medication administration can affect blood pressure control. In patients with uncontrolled hypertension on 3 or more antihypertensive medications all taken on awakening, switching one medication to bedtime administration resulted in significantly reduced 24-hour systolic and diastolic blood pressures, with $21.7 \%$ to $37 \%$ of those who changed one medication to bedtime dosing having their blood pressures subsequently controlled. ${ }^{38,39}$ Also shown was a reduction in the nocturnal nondipping pattern $(<10 \%$ reduction in nocturnal versus daytime blood pressures), which may be of relevance given the evidence showing that nocturnal blood pressure 
Table 2 History and physical examination elements suggestive of secondary causes of hypertension 1,3,14,19,29,33,70

\begin{tabular}{|c|c|c|c|}
\hline Disease & Historical findings & Physical exam findings & $\begin{array}{l}\text { Laboratory or diagnostic study } \\
\text { findings }\end{array}$ \\
\hline Chronic kidney disease & $\begin{array}{l}\text { Comorbidities potentially } \\
\text { causing kidney damage } \\
\text { Nocturia }\end{array}$ & Edema & $\begin{array}{l}\text { Decreased creatinine clearance, } \\
\text { abnormal urinalysis (proteinuria, } \\
\text { hematuria, pyuria), abnormal renal } \\
\text { ultrasonography, MRA, CTA, or ACE } \\
\text { inhibitor renal scan }\end{array}$ \\
\hline Coarctation of the aorta & & $\begin{array}{l}\text { Differential in brachial } \\
\text { and femoral pulses, } \\
\text { systolic bruit, systolic } \\
\text { heart murmur }\end{array}$ & $\begin{array}{l}\text { Echocardiography findings consistent } \\
\text { with coarctation }\end{array}$ \\
\hline Cushing's syndrome & $\begin{array}{l}\text { Muscle weakness or fatigue, } \\
\text { emotional disturbances, } \\
\text { decreased libido, amenorrhea }\end{array}$ & $\begin{array}{l}\text { Moon facies, } \\
\text { central adiposity, } \\
\text { abdominal striae, } \\
\text { interscapular fat deposition, } \\
\text { fluid retention }\end{array}$ & $\begin{array}{l}\text { Elevated plasma cortisol level after } \\
\text { dexamethasone administration }\end{array}$ \\
\hline Obstructive sleep apnea & $\begin{array}{l}\text { Snoring, witnessed } \\
\text { apnea, excessive daytime } \\
\text { somnolence, male gender }\end{array}$ & $\begin{array}{l}\text { Obese, redundant pharyngeal } \\
\text { soft, tissues, large shirt collar } \\
\text { size }\end{array}$ & Abnormal sleep study \\
\hline Pheochromocytoma & $\begin{array}{l}\text { Episodic hypertension with } \\
\text { diaphoresis, palpitations, or } \\
\text { headache, positive family } \\
\text { history, labile blood } \\
\text { pressure }\end{array}$ & $\begin{array}{l}\text { Café-au-lait spots or } \\
\text { neurofibromas, } \\
\text { suprarenal or midline } \\
\text { abdominal mass }\end{array}$ & $\begin{array}{l}\text { Elevated } 24 \text {-hour urine metanephrine } \\
\text { to creatinine ratio or plasma free } \\
\text { metanephrine ( } 90 \%-100 \% \text { sensitive } \\
\text { and } 89 \%-97.6 \% \text { specific) }\end{array}$ \\
\hline Primary aldosteronism & $\begin{array}{l}\text { Muscle cramps } \\
\text { Weakness } \\
\text { Polyuria } \\
\text { Polydipsia (less common) }\end{array}$ & & $\begin{array}{l}\text { Elevated aldosterone/renin ratio, } \\
\text { elevated serum aldosterone with salt } \\
\text { loading, hypokalemia (usually a later } \\
\text { finding), abnormal CT scan showing } \\
\text { adrenal adenoma }\end{array}$ \\
\hline Renal artery stenosis & $\begin{array}{l}\text { Young female (fibromuscular } \\
\text { disease), older age, smoker, } \\
\text { history of atherosclerotic } \\
\text { disease, renal insufficiency, } \\
\text { absence of obesity, history } \\
\text { of flash pulmonary edema } \\
\text { (atheroscleric lesion) }\end{array}$ & Renal or carotid artery bruit & $\begin{array}{l}\text { Impaired renal function after addition } \\
\text { of or increased dose of ACE inhibitor } \\
\text { or angiotensin-receptor blocker }\end{array}$ \\
\hline
\end{tabular}

Abbreviations: MRA, magnetic resonance angiography; CTA, computer tomographic angiography; ACE, angiotensin-converting enzyme.

readings are a better predictor of cardiovascular complications than 24-hour or daytime blood pressure readings. ${ }^{40-43}$

In a small study of 44 Brazilian patients, adding a pharmacist to the health care delivery team demonstrated improvement in blood pressure reduction, although half of the patients still had not reached their blood pressure goal, and showed improvement in one measure of social functioning. ${ }^{44}$ Pharmacist interventions included patient education, blood pressure measurement, and assessing adherence. Patient medication adherence increased from $63.3 \%$ to $95.5 \%$ at study end.

\section{Pharmacologic treatment}

Pharmacologic treatment should concentrate on use of an appropriate diuretic with a thiazide diuretic for most patients and a loop diuretic for those with a decreased glomerular filtration rate. ${ }^{10}$ One recommended treatment foundation is a combination of a thiazide diuretic with a long-acting calcium channel blocker (CCB) and an ACE inhibitor or an angiotensin receptor blocker. ${ }^{10}$ Chlorthalidone may be preferred over hydrochlorothiazide, particularly in patients with resistant hypertension (see Table 4). ${ }^{24}$ Additional fourth-, fifth-, and sixth-line agents can be added based on individual patient characteristics with consideration given to treatment recommendations outlined by JNC VII. ${ }^{36}$ Other agents which should be considered include a mineralocorticoid antagonist, a combination alpha-beta blocker over a pure beta blocker, or direct vasodilating agents (hydralazine or minoxidil). ${ }^{10}$ 
Table 3 Treatment of secondary causes of hypertension ${ }^{3,19,29}$

\begin{tabular}{ll}
\hline Disease & Treatment recommendations \\
\hline Chronic kidney disease & Diuretic treatment (loop diuretic preferred with decreased glomerular filtration rate), \\
& ACE inhibitors to decrease proteinuria and slow progression of diabetic nephropathy, \\
calcium channel blocker & Surgery or balloon angioplasty \\
Coarctation of the aorta & Surgical resection of pituitary adenoma, pituitary irradiation \\
Cushing's disease & CPAP, weight loss, consider aldosterone antagonists \\
Obstructive sleep apnea & Surgery, $\alpha$ - and/or $\beta$-blockers \\
Pheochromocytoma & Surgical removal of adrenal adenoma, aldosterone antagonists for adrenal hyperplasia, \\
Primary aldosteronism & non-surgical candidates \\
& Controversial, ongoing trial (CORAL) evaluating medical treatment vs stent placement \\
Renal artery stenosis - atherosclerotic disease & Stent preferred over balloon angioplasty \\
Renal artery stenosis - fibromuscular disease & Balloon angioplasty \\
\hline
\end{tabular}

Abbreviations: CPAP, continuous positive airway pressure; $\mathrm{ACE}$, angiotensin-converting enzyme.

\section{Diuretics}

Diuretic treatment is an important component of pharmacologic management of resistant hypertension since hypervolemia is common in patients with RH. ${ }^{19,24,45}$ Hypervolemia can result from excess sodium ingestion, progressive renal damage, fluid retention from blood pressure reduction, and underuse of diuretic treatment. ${ }^{18}$ Diuretics are underused in the treatment of hypetension ${ }^{1,37,46}$ for a variety of reasons, including concern regarding metabolic side effects and misconceptions about maximum dosages. ${ }^{3,36}$ Addition of, increasing the dose of, or changing the class of diuretic is a recommended first step in the treatment approach to $\mathrm{RH}^{24}$ and may improve blood pressure in up to $50 \%$ of patients. ${ }^{19,46}$

Hydrochlorothiazide is used more commonly in clinical practice $^{13}$ than chlorthalidone, a thiazide-like diuretic, probably because of increased ease of use with hydrochlorothiazide having more formulations available and being available in fixed-dose combination pills. ${ }^{37,45,46}$ While chlorthalidone and hydrochlorothiazide have not been compared for efficacy or tolerability in head-to-head trials, the greater clinical trial evidence is found for use of chlorthalidone for the prevention of cardiovascular morbidity and mortality. ${ }^{45}$ Hydrochlorothiazide is commonly used in doses up to $25 \mathrm{mg}$ daily, however doses up to $50 \mathrm{mg}$ daily can provide improved control. ${ }^{36}$ Despite similar office blood pressure readings, chlorthalidone provides more effective 24-hour blood pressure control than hydrochlorothiazide. ${ }^{47}$ This may be explained by their differences in duration of action: 16 to 24 hours for hydrochlorothiazide and 48 to 72 hours for chlorthalidone..$^{48}$ Chlorthalidone is approximately twice as potent as hydrochlorothiazide; eg, $25 \mathrm{mg}$ of hydrochlorothiazide is roughly equipotent to $12.5 \mathrm{mg}$ of chlorthalidone. ${ }^{47,48}$ Hypokalemia has been raised as a concern with chlorthalidone. One cross-over study found no difference in the incidence of hypokalemia between equipotent dosages of hydrochlorothiazide and chlorthalidone, ${ }^{47}$ but overall the evidence is mixed about the incidence of hypokalemia between the two agents. ${ }^{13,46}$ Given its better 24-hour blood pressure control, chlorthalidone up to $25 \mathrm{mg}$ daily is recommended preferentially in resistant hypertension. ${ }^{1,10,45,46,49}$ Caution should be used with thiazide diuretics in patients with a history of significant hyponatremia, hypokalemia or gout. ${ }^{36}$ Sodium and potassium levels should be monitored regardless of choice of diuretic. ${ }^{45}$

For patients with decreased renal function (creatinine clearance $<30$ to $50 \mathrm{~mL} / \mathrm{min}$ ), thiazide diuretics are not as effective. ${ }^{1,3}$ Therefore, for these patients, loop diuretics are preferred. If short-acting loop diuretics such as furosemide or bumetanide are used, they must be dosed at least twice daily for effective blood pressure control.,38,50

\section{Aldosterone antagonists}

Spironolactone, an aldosterone-antagonist, is an important tool in the pharmacologic management of resistant hypertension. As mentioned above, aldosterone excess is common in patients with resistant hypertension and also appears to be related to obesity and obstructive sleep apnea, two prevalent co-morbidities among patients with resistant hypertension. Therefore, it is logical that aldosterone blockade would play a role in the treatment of resistant hypertension. Studies demonstrate a benefit of spironolactone treatment in patients both with and without primary aldosteronism. ${ }^{28}$

The addition of low dose spironolactone (range of 12.5 to $50 \mathrm{mg}$ daily) in the setting of refractory or resistant hypertension lowered systolic blood pressure by 21.7 to $28 \mathrm{mmHg}$ 


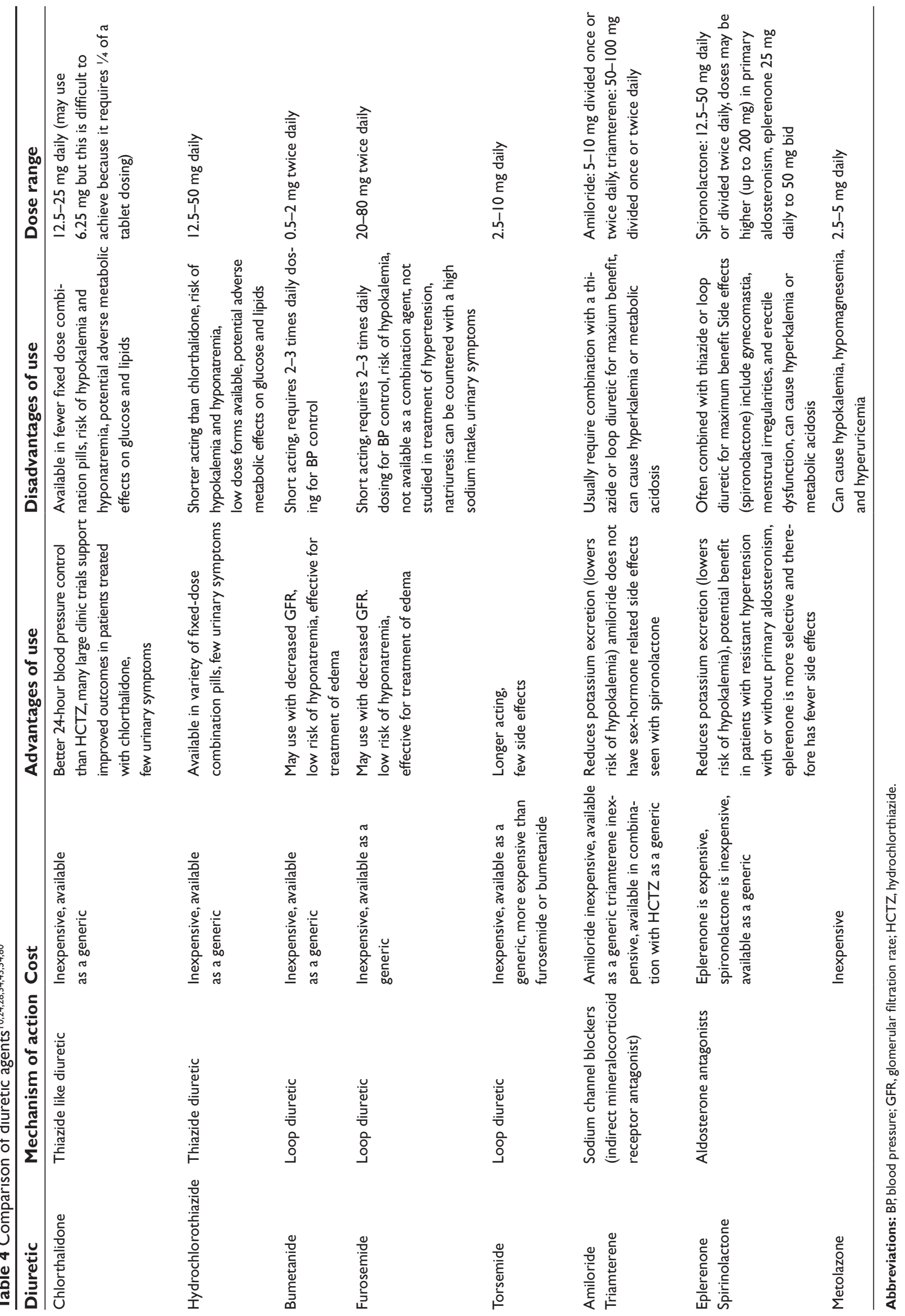


and diastolic blood pressure by 8.5 to $13 \mathrm{mmHg}$. This was achieved regardless of presence of primary aldosteronism. In addition, pretreatment serum aldosterone levels, aldosterone excretion, and aldosterone/renin ratio did not predict blood pressure response to spironolactone. ${ }^{51-55}$ This has lead to increased recommendation for spironolactone in resistant hypertension, ${ }^{1,9,10,28,51-55}$ even as a fourth-line agent. ${ }^{13,44}$

Dosing is usually initiated at $25 \mathrm{mg}$ daily, though $12.5 \mathrm{mg}$ daily may be more appropriate in patients with diabetes mellitus, chronic kidney disease, and in the elderly. Spironolactone can be initiated simultaneously with a thiazide diuretic for the dual benefit of blood pressure control and potassium balance. ${ }^{13,28,56}$ Those on ACE inhibitors or ARBs are at increased risk of hyperkalemia. If patients are taking supplemental potassium, this should be discontinued (or reduced if taking significant quantities) on initiation of spironolactone. Potassium levels and renal function should be assessed in 4 weeks after initiation, except for the high risk groups mentioned previously, where assessment sooner may be warranted. Titration should be at 4- to 6-week intervals, with repeat assessment of potassium and renal function as above. Mild hyperkalemia can usually be managed with a reduction in dose, while with serum potassium levels $>5.5 \mathrm{mEq} / \mathrm{L}$, spironolactone should be discontinued, possibly resuming at a lower dose once potassium levels have normalized. Dosages up to $50 \mathrm{mg}$ daily have been studied in resistant hypertension; higher dosages may be warranted in those with confirmed primary aldosteronism. ${ }^{54,57,58}$

In addition to hyperkalemia and renal insufficiency, breast tenderness is a commonly cited adverse reaction to spironolactone. This effect is dose dependent and occurs in approximately $10 \%$ of men taking $25 \mathrm{mg}$ daily. ${ }^{28,54}$ In these patients, therapy can be discontinued, then restarted at a lower dose once the tenderness has resolved. Other adverse reactions include gynecomastia, menstrual irregularities, and erectile dysfunction. ${ }^{54,58}$

Amiloride, an indirect aldosterone antagonist that is better tolerated due to its lack of antiandrogenic action, has not been studied in resistant hypertension as much as spironolactone has. One small study found that in patients with uncontrolled hypertension, the addition of $2.5 \mathrm{mg}$ of amiloride daily decreased systolic blood pressure by $31 \mathrm{mmHg}$ and diastolic by $15 \mathrm{mmHg} .{ }^{59}$ However, an audit of the use of $10 \mathrm{mg}$ amiloride showed half the blood pressure reduction of spironolactone $25 \mathrm{mg}$ daily. ${ }^{60}$ Until more studies are completed, spironolactone should be used preferentially, unless the patient is unable to tolerate spironolactone. If amiloride is used, monitoring of potassium and renal function should be performed as mentioned above for spironolactone.
Eplerenone, a more selective aldosterone antagonist, is effective in the treatment of hypertension but has not been well studied in RH. ${ }^{10,13}$ It may be considered in patients intolerant of spironolactone, particularly those with sex-hormone related side effects (breast tenderness, gynecomastia, erectile dysfunction, and menstrual irregularities). ${ }^{24}$

\section{Renin-angiotensin blockade}

ACE inhibitors also have a long history of use for hypertension and are particularly recommended in patients with diabetes mellitus, heart failure, post-myocardial infarction, chronic kidney disease, high coronary disease risk, and recurrent stroke prevention. ${ }^{36,61}$ ARBs are an alternative for patients intolerant of ACE inhibitors. While studies looking at the combination of ACE inhibitors and ARBs in treating hypertension have shown improvement compared with monotherapy, most of these studies have not used maximum dosing of the monotherapy agent. ${ }^{1}$ In addition, combination ACE inhibitor/ARB therapy is not as effective as adding a diuretic or $\mathrm{CCB}$ to an $\mathrm{ARB} .{ }^{62}$ Recent studies have indicated the possibility of increased adverse renal outcomes with dual ACE inhibitor and ARB treatment while not improving other clinical outcomes. ${ }^{63,64}$ Thus, dual ACE inhibitor and ARB therapy is not currently recommended for most patients. ${ }^{1,50,65}$ Dosage of an ACE inhibitor or ARB may be increased to the maximum recommended dosage as long as the serum creatinine does not increase more than $35 \%$ above baseline and hyperkalemia does not develop. ${ }^{36}$ Some patients will develop a mild ( 0.4 to $0.6 \mathrm{mmol} / \mathrm{dL})$ increase in serum potassium levels that is self limiting. ${ }^{66}$

\section{Calcium channel blockers}

CCBs are further divided into dihydropyridine CCBs (amlodipine, felodipine, nicardipine, nifedipine, nisoldipine) and nondihydropyridine CCBs (diltiazem and verapamil). Both are recommended as possible initial drug therapy for hypertension by JNC VII. ${ }^{36}$ Dihydropyridine CCBs bind with greater affinity to blood vessel receptors compared with nondihydropyridine CCBs which have equal affinity for blood vessel and cardiac receptors. ${ }^{67}$ In doses used in clinical practice, nondihydropyridine CCBs can cause decreased cardiac conduction and myocardial contractility. ${ }^{67} \mathrm{CCBs}$ have been recommended as a component of a three-medication regimen which includes a thiazide diuretic and either an ACE inhibitor or an ARB, based partly on their complementary mechanism of action. ${ }^{1,50}$ Common side effects of CCBs include constipation and edema, the later usually not responsive to diuretic therapy. ${ }^{46}$ 


\section{$\beta$-blockers and $\alpha$-blockers}

$\beta$-blockers have long been used for hypertension and are particularly recommended for patients with increased cardiovascular disease risk, heart failure, post-myocardial infarction, and diabetes mellitus. ${ }^{36}$ While masking of hypoglycemia has been a concern of using $\beta$-blockers in the setting of diabetes mellitus, a review found no evidence of $\beta_{1}$-selective blockers masking hypoglycemia, prolonging hypoglycemia, or adversely affecting glucose metabolism. ${ }^{68} \beta$-blockers do have a disadvantage of reflexively causing increased peripheral edema. This has lead some experts to recommend $\alpha$-blockers in conjunction with $\beta$-blockade, though not as first line therapy. ${ }^{1,3,13,49}$ This is in part due to the significant increase in cardiovascular events and hospitalization in doxazosin compared with chlorthalidone seen in the ALLHAT study that resulted in the early termination of the doxazosin arm. ${ }^{69}$ That said, $\alpha$-blockers may be more a more logical choice in patients with lower urinary tract symptoms. Particular care should be taken to monitor for orthostatic hypotension with the use of $\alpha$-blockers.

\section{Combination strategies}

Thiazide diuretics can be safely and effectively combined with almost all classes of antihypertensive medications. ${ }^{24}$ A three-drug regimen containing a thiazide diuretic, a calcium-channel blocker, and an ACE inhibitor or ARB is advocated. ${ }^{24,28}$ While this triple therapy has not been widely studied, thiazide diuretics in combination with most classes of medications have proven more effective in blood pressure lowering than combinations not containing a diuretic. ${ }^{24}$

No specific combination regimen has been shown superior to others in the treatment of resistant hypertension. However, it is logical to use certain principles in guiding combination treatment. Patients should be treated with antihypertensive agents per compelling indications. ${ }^{36}$ Aside from these recommendations, patients can be assessed for the presumed underlying mechanism of hypertension (reninbased, aldosterone-based, hemodynamic/volume) in order to guide therapy recognizing that physician assessment of the underlying cause is both complex and difficult. ${ }^{14,70}$ What may be more practical is to assess the patient's current regimen and treatment response, as most patients with resistant hypertension are already on a number of different agents. Patients with an incomplete response to a certain agent may benefit from an increased dose of that agent or addition of a second agent with a complimentary mechanism of action. ${ }^{13}$ While substitution of one agent for another is a potential strategy, add-on therapy is generally preferred, ${ }^{13}$ since complimentary mechanisms of action will improve overall efficacy of the regimen.

When adding on medications to combinations already containing a diuretic, $\mathrm{CCB}$, ACE inhibitor or ARB, consideration should be given to combined $\alpha-\beta$ blockers over pure $\beta$-blockers ${ }^{3,71}$ and either centrally acting medications (clonidine, methyldopa) or direct vasodilators (minoxidil, hydralazine) although use of these latter two classes is limited by tolerability and frequent dosing. ${ }^{24}$ Combined $\alpha-\beta$ blockers, either through use of a single agent (carvedilol, labetalol) or two agents may offer benefits particularly in patients who have failed to achieve target blood pressure on a diuretic/ACE-inhibitor combination. ${ }^{71}$ As a result of the potent vasodilation produced by direct acting vasodilators, $\beta$-blockers and loop diuretics are usually necessary to overcome the reflex tachycardia and fluid retention, respectively. If patients are requiring these medications to control their blood pressure, hypertension specialists should likely be involved. ${ }^{1,13,49,72}$

Fixed-dose combination pills have been shown to increase adherence versus equivalent therapy with single-agent medications ${ }^{73}$ however few studies have been performed comparing the efficacy of combination pills versus using combined monotherapy agents from each class included in the fixed-dose combination pill. ${ }^{74}$ Unfortunately, most fixed dose combination pills do not provide maximum dosages of included agents, ${ }^{74}$ though many monotherapy agents will require multiple pills to reach maximum dosages as well.

\section{Other agents}

Endothelin receptor antagonists are a new family of antihypertensive medications that are currently being evaluated. Darusentan, a selective endothelin receptor antagonist currently under investigation and not yet available for clinical use, demonstrated sustained dose-dependent lowering of blood pressure in phase II studies. ${ }^{75}$ Darusentan is an antagonist selective for type A endothelin receptors, activation of which causes vasoconstriction and proliferation of vascular smooth muscle. ${ }^{75}$ Medication interactions, though not tested, are theorized to be low based on its elimination profile. ${ }^{76}$ Doses of up to $300 \mathrm{mg}$ daily of darusentan were associated with significant reductions in both systolic and diastolic blood pressures that persisted over 24 hours, as measured by ambulatory blood pressure monitoring. However, there was no statistically significant difference between the groups in percent of patients achieving blood pressure goals (although there was a trend favoring darusentan) $\cdot{ }^{75}$ Headache and edema were the most common intervention related side effects. ${ }^{75}$ Potential adverse events of 
endothelin receptor antagonists (teratogenicity, hepatotoxicity) limit their use as first-line agents, but this study indicates a possible role for darusentan in the treatment of resistant hypertension. It should be noted that significant hepatotoxicity was not observed in this 10 -week trial study. ${ }^{75}$

A double blind placebo-controlled randomized trial of obese patients with uncontrolled hypertension despite treatment found that orlistat, compared with placebo, resulted in decreased diastolic blood pressures $(-11.4 \mathrm{mmHg}$ compared with $-9.2 \mathrm{mmHg}$ ) and improved rates of achieving diastolic blood pressure goals (67\% compared to $53 \%) .{ }^{77}$ The group treated with orlistat did achieve greater weight loss than the placebo group. The study was limited by high drop out rates in both the orlistat and placebo arm.

For patients who are truly resistant to antihypertensive medications and a thorough work-up for secondary causes has been completed, electrical carotid sinus baroreflex stimulation implantable devices have been shown in feasibility trials to reduce systolic blood pressure by 22 to $24 \mathrm{mmHg} .^{78}$

\section{Conclusion}

Resistant hypertension should be differentiated from uncontrolled hypertension due to medication nonadherence, undertreatment, or pseudoresistance. True resistant hypertension can be diagnosed by taking a careful history, through confirmatory blood pressure measurement with 24-hour ambulatory blood pressure monitoring, and by detecting clues on examination that indicate the presence of end-organ damage. When resistant hypertension is diagnosed, it is important to consider secondary causes such as primary aldosteronism or obstructive sleep apnea that may be causing or contributing to elevated blood pressure readings. Treatment is focused on both nonpharmacologic (weight loss, exercise, limiting sodium intake) and pharmacologic modalities. Additionally, identification of and treatment of secondary causes is essential. Pharmacologic treatment initially focuses on optimizing diuretic treatment and is followed by selection of patient specific combination strategies. Referral to a hypertension specialist is considered when pharmacologic treatment fails to achieve blood pressure goals.

\section{Disclosures}

The authors have no conflicts of interest to disclose.

\section{References}

1. Calhoun DA, Jones D, Textor S, et al. Resistant hypertension: diagnosis, evaluation, and treatment: a scientific statement from the American Heart Association Professional Education Committee of the Council for High Blood Pressure Research. Circulation. 2008;117(25):e510-e526.

2. Epstein M. Resistant hypertension: prevalence and evolving concepts. J Clin Hypertens (Greenwich). 2007;9(1 Suppl 1):2-6.
3. Moser M, Setaro JF. Resistant or difficult-to-control hypertension. N Engl J Med. 2006;355(4):385-392.

4. Cuspidi C, Macca G, Sampeiri L, et al. High prevalence of cardiac and extracardiac target organ damage in refractory hypertension. J Hypertens. 2001;19(11):2063-2070.

5. Pierdomenico SD, Lapenna D, Bucci A, et al. Cardiovascular outcome in treated hypertensive patients with responder, masked, false resistant, and true resistant hypertension. Am J Hypertens. 2005;18(11):1422-1428.

6. Erdine S, Arat-Ozkan A. Resistant Hypertension. Blood Press. 2003;12(5-6):347-348.

7. Calhoun DA, Nishikaza MK, Zaman A, Thakkar RB, Weissmann P. Hyperaldosteronism among black and white subjects with resistant hypertension. Hypertension. 2002;40(6):892-896.

8. Gaddam KK, Nishizaka MK, Pratt-Ubunama M, et al. Characterization of resistant hypertension: association between resistant hypertension, aldosterone, and persistent intravascular volume expansion. Arch Intern Med. 2008;168(11):1159-1164.

9. Pimenta E, Gaddam KK, Oparil S. Mechanisms and treatment of resistant hypertension. J Clin Hypertens (Greenwich). 2008;10(3):239-244.

10. Calhoun DA. Low-dose aldosterone blockade as a new treatment paradigm for controlling resistant hypertension. J Clin Hypertens (Greenwich). 2007;9(1 Suppl 1):19-24.

11. Brown MA, Buddle ML, Martin A. Is resistant hypertension really resistant? Am J Hypertens. 2001;14(12):1263-1269.

12. Park J, Campese V. Clinical characteristics of resistant hypertension: the importance of compliance and the role of diagnostic evaluation in delineating pathogenesis. J Clin Hypertens (Greenwich). 2007;9(1 Suppl 1):7-12.

13. Trewet CLB, Ernst ME. Resistant hypertension: identifying causes and optimizing treatment recommendations. Southern Med J. 2008;101(2):166-173.

14. Vidt DG. Pathogenesis and treatment of resistant hypertension. Minerva Med. 2003;94(4):201-214.

15. Pickering TG. Arterial stiffness as a cause of resistant hypertension? J Clin Hypertens (Greenwich). 2007;9(5):390-395.

16. Muxfeldt ES, Bloch KV, da Rocha Nogueira A, Salles GH. True resistant hypertension: is it possible to be recognized in the office? Am J Hypertens. 2005;18(12):1534-1540.

17. Hall JE. The kidney, hypertension, and obesity. Hypertension. 2003; 41[part 2]:625-633.

18. Kaplan NM. Resistant hypertension. J Hypertens. 2005;23(8):1441-1444.

19. Hall WD. Resistant hypertension, secondary hypertension, and hypertensive crises. Cardiol Clin. 2002(2);20:281-289.

20. Cadaval Goncalves S, Martinez D, Gus M, et al. Obstructive sleep apnea and resistant hypertension: a case-control study. Chest. 2007;132(6): 1858-1862.

21. Pratt-Ubunama MN, Nishizaka MK, Boedefeld RL, Cofield SS, Harding SM, Calhoun DA. Plasma aldosterone is related to severity of obstructive sleep apnea in subjects with resistant hypertension. Chest. 2007;131(2):453-459.

22. Calhoun DA, Nishizaka MK, Zaman MA, Harding SM. Aldosterone excretion among subjects with resistant hypertension and symptoms of sleep apnea. Chest. 2004;125(1):112-117.

23. Douma S, Petidis K, Doumas M, et al. Prevalence of primary aldosteronism in resistant hypertension: a retrospective observational study. Lancet. 2008;371(9628):1921-1926.

24. Epstein M, Calhoun DA. The role of aldosterone in resistant hypertension: implications for pathogenesis and therapy. Curr Hypertens Rep. 2007;9(2):98-105.

25. DuprezDA. Aldosterone and the vasculature: mechanisms mediating resistant hypertension. J Clin Hypertens (Greenwich). 2007;9(1 Suppl 1):13-18.

26. Grim CE. Evolution of diagnostic criteria for primary aldosteronism: why is it more common in "drug-resistant" hypertension today? Curr Hypertens Rep. 2004;6(6):485-492.

27. Sartori M, Calo LA, Mascagna V, et al. Aldosterone and refractory hypertension: a prospective cohort study. Am J Hypertens. 2006;19(4):373-379. 
28. Pimenta E, Calhoun DA. Resistant hypertension and aldosteronism. Curr Hypertens Rep. 2007;9(5):353-359.

29. Pimenta E, Calhoun DA, Oparil S. Mechanisms and treatment of resistant hypertension. Arq Bras Cardiol. 2007;88(6):604-613.

30. Campese VM, Mitra N, Sandee D. Hypertension in renal parenchymal disease: why is it so resistant to treatment? Kidney Int. 2006:69(6):967-973.

31. Burnier M, Santschi V, Favrat B, Brunner HR. Monitoring compliance in resistant hypertension: an important step in patient management. J Hypertens. 2003;21(Suppl 2):S37-S42.

32. Pickering TG, Hall JE, Appel LJ, et al. Recommendations for blood pressure management in humans and experimental animals: a statement for professionals from the Subcommittee of Professional and Public Education of the American Heart Association Council on High Blood Pressure Research. Circulation. 2005;111(5):697-716.

33. Hickman PE, Leong M, Chang J, Wilson SR, McWhinney B. Plasma free metanephrines are superior to urine and plasma catecholamines and urine catecholamine metabolites for the investigation of phaeochromocytoma. Pathology. 2009;41(2):173-177.

34. Cuspidi C, Meani S, Valerio C, Fusi V, Sala C, Zanchetti A. Left ventricular hypertrophy and cardiovascular risk stratification: impact and cost-effectiveness of echocardiography in recently diagnosed essential hypertensives. J Hypertens. 2006;24(8):1671-1677.

35. Kawashima A, Francis IR, Baumgarten DA, et al; for the Expert Panel on Urologic Imaging. Renovascular hypertension. [online publication]. Reston (VA): American College of Radiology (ACR); 2007. p. 9.

36. Chobanian AV, Bakris GL, Black HR, et al. The Seventh Report of the Joint National Committee on Prevention, Detection, Evaluation, and Treatment of High Blood Pressure: the JNC 7 report. JAMA. 2003;289(19):2560-2572.

37. Calhoun DA. Resistant or difficult-to-control hypertension. J Clin Hypertens (Greenwich). 2006;8(3):181-186.

38. Hermida RC, Ayala DE, Calvo C, et al. Effects of time of day of treatment on ambulatory blood pressure pattern of patients with resistant hypertension. Hypertension. 2005;46(4):1053-1059.

39. Hermida RC, Ayala DE, Fernandez JR, Calvo C. Chronotherapy improves blood pressure control and reverts the nondipper pattern in patients with resistant hypertension. Hypertension. 2008;51(1):69-76.

40. Dolan E, Stanton A, Thijs L, et al. Superiority of ambulatory over clinic blood pressure measurement in predicting mortality: the Dublin outcome study. Hypertension. 2005;46(1):156-161.

41. Kikuya M, Ohkubo T, Asayama K, et al. Ambulatory blood pressure and 10-year risk of cardiovascular and noncardiovascular mortality: the Ohasama study. Hypertension. 2005;45(2):240-245.

42. Staessen JA, Thijs L, Fagard R, et al. for the Systolic Hypertension in Europe Trial Investigators . Predicting cardiovascular risk using conventional vs. ambulatory blood pressure in older patients with systolic hypertension. JAMA. 1999;282(6):539-546.

43. Brotman DJ, Davidson MB, Boumitri M, Vidt DG. Impaired diurnal blood pressure variation and all-cause mortality. Am J Hypertens. 2008;21(1):92-97.

44. De Souza WA, Yugar-Toledo JC, Bergsten-Mendes G, Sabha M, Moreno H. Effect of pharmaceutical care on blood pressure control and health-related quality of life in patients with resistant hypertension. Am J Health-Syst Pharm. 2007;64(18):1955-1961.

45. Taler SJ. Should chlorthalidone be the diuretic of choice for antihypertensive therapy? Curr Hypertens Rep. 2008;10(4):293-297.

46. Handler J. Maximizing diuretic therapy in resistant hypertension. J Clin Hypertens (Greenwich). 2007;9(10):802-806.

47. Ernst ME, Carter BL, Goerdt CJ, et al. Comparative antihypertensive effects of hydrochlorothiazide and chlorthalidone on ambulatory and office blood pressure. Hypertension. 2006;47(3):352-358.

48. Carter BL, Ernst ME, Cohen JD. Hydrochlorothiazide versus chlorthalidone: evidence supporting their interchangeability. Hypertension. 2004;43(1):4-9.

49. Moser M, Cushman W, Handler J. Resistant or difficult-to-treat hypertension. J Clin Hypertens (Greenwich). 2006;8(6):434-440.

50. Sarafidis PA, Bakris GL. Resistant hypertension: an overview of evaluation and treatment. J Am Coll Cardiol. 2008;52(22):1749-1757.
51. Chapman N, Dobson J, Wilson S, et al; for Anglo-Scandinavian Cardiac Outcomes Trial Investigators. Effect of spironolactone on blood pressure in subjects with resistant hypertension. Hypertension. 2007;49(4):839-845.

52. Lane DA, Shah S, Beevers DG. Low-dose spironolactone in the management of resistant hypertension: a surveillance study. J Hypertens. 2007;25(4):891-894.

53. Mahmud A, Mahgoub M, Hall M, Feely J. Does aldosterone-torenin ratio predict the antihypertensive effect of the aldosterone antagonist spironolactone? Am J Hypertens. 2005;18(12 Pt 1): 1631-1635.

54. Nishizaka MK, Zaman MA, Calhoun DA. Efficacy of low-dose spironolactone in subjects with resistant hypertension. Am J Hypertens. 2003;16(11 Pt 1):925-930.

55. Ouzan J, Perault C, Lincoff AM, Carre E, Mertes M. The role of spironolactone in the treatment of patients with refractory hypertension. Am J Hypertens. 2002;15(4 Pt 1):333-339.

56. Nishkzaka MK, Calhound DA. The role of aldosterone antagonists in the management of resistant hypertension. Curr Hypertens Rep. 2005;7(5):343-347.

57. Calhoun DA. Use of aldosterone antagonists in resistant hypertension. Prog Cardiovasc Dis. 2006;48(6):387-396.

58. Gaddam KK, Pratt-Ubunama MN, Calhoun DA. Aldosterone antagonists: effective add-on therapy for the treatment of resistant hypertension. Expert Rev Cardiovasc Ther. 2006;4(3):353-359.

59. Eide IK, Torjesen PA, Drolsum A, Babovic A, Lilledahl NP. Low-renin status in therapy-resistant hypertension: a clue to efficient treatment. J Hypertens. 2004;22(11):2217-2226.

60. Lane DA, Beevers DG. Amiloride $10 \mathrm{mg}$ is less effective than spironolactone $25 \mathrm{mg}$ in patients with hypertension resistant to a multidrug regimen including an angiotensin-blocking agent. $J$ Hypertens. 2007;25(12):2515-2516.

61. Dora JM, Kramer CK, Canani LH. Standards of Medical Care in Diabetes - 2008: response to Hirsch, Inzucchi, and Kirkman. Diabetes Care. 2008;31(5):e44; author reply e5.

62. Stergiou GS, Makris T, Papavasiliou M, Efstathiou S, Manolis A. Comparison of antihypertensive effects of an angiotensin-converting enzyme inhibitor, a calcium antagonist and a diuretic in patients with hypertension not controlled by angiotensin receptor blocker monotherapy. J Hypertens.2005;23(4):883-889.

63. Yusuf S, Teo KK, Pogue J, et al. Telmisartan, ramipril, or both in patients at high risk for vascular events. The New England journal of medicine 2008;358(15):1547-1559.

64. Pfeffer MA, McMurray JJ, Velazquez EJ, et al. Valsartan, captopril, or both in myocardial infarction complicated by heart failure, left ventricular dysfunction, or both. $N$ Engl $J$ Med. 2003;349(20):1893-1906.

65. Arici M, Erdem Y. Dual blockade of the renin-angiotensin system for cardiorenal protection: an update. Am J Kidney Dis. 2009;53(2): 332-345.

66. Bakris GL, Weir MR. Angiotensin-converting enzyme inhibitor-associated elevations in serum creatinine: is this a cause for concern? Arch Intern Med. 2000;160(5):685-693.

67. Muntwyler J, Follath F. Calcium channel blockers in treatment of hypertension. Prog Cardiovasc Dis. 2001;44(3):207-216.

68. Sawicki PT, Siebenhofer A. Betablocker treatment in diabetes mellitus. J Intern Med. 2001;250(1):11-17.

69. SoRelle R. National Heart, Lung, and Blood Institute halts part of antihypertensive and lipid-lowering treatment to prevent heart attack trial (ALLHAT). Circulation. 2000;101(12):E9025.

70. Taler SJ. Treatment of resistant hypertension. Curr Hypertens Rep. 2005; 7(5):323-329.

71. Mann SJ. Combined alpha/beta-blockade: an underused approach to the treatment of resistant hypertension. J Clin Hypertens. 2007;9(9): 663-664.

72. Sica DA. Minoxidil: an underused vasodilator for resistant or severe hypertension. J Clin Hypertens (Greenwich). 2004;6(5):283-287. 
73. Jackson KC, 2nd, Sheng X, Nelson RE, Keskinaslan A, Brixner DI. Adherence with multiple-combination antihypertensive pharmacotherapies in a US managed care database. Clinical therapeutics. 2008;30(8):1558-1563.

74. Chrysant SG. Using fixed-dose combination therapies to achieve blood pressure goals. Clin Drug Investig. 2008;28(11):713-734.

75. Black HR, Bakris GL, Weber MA, et al. Efficacy and safety of darusentan in patients with resistant hypertension: results from a randomized, double-blind, placebo-controlled dose-ranging study. J Clin Hypertens (Greenwich). 2007;9(10):760-769.

76. Epstein BJ. Efficacy and safety of darusentan: a novel endothelin receptor antagonist. Ann Pharmacother. 2008;42(7):1060-1069.
77. Bakris G, Calhoun D, Ega, B, et al; for orlistat and resistant hypertension investigators. Orlistat improves blood pressure control in obese subjects with treated but inadequately controlled hypertension. J Hypertens. 2002;20(11):2257-2267.

78. Sloand JA, Illig KA, Bisognano JD. Improved control of resistant hypertension with device-mediated electrical carotid sinus baroreflex stimulation. J Clin Hypertens (Greenwich). 2007;9(9):716-719.

79. Gyamlani G, Geraci SA. Secondary hypertension due to drugs and toxins. Southern Med J. 2007;100(7):692-699.

80. Padilla MCA, Armas-Hernandez MJ, Hernandez RH, Israili ZH, Valasco M. Update of diuretics in the treatment of hypertension. Am J Ther. 2007;14(2):154-160.

\section{Publish your work in this journal}

Integrated Blood Pressure Control is an international, peer-reviewed open-access journal focusing on the integrated approach to managing hypertension and risk reduction. Treating the patient and comorbidities together with diet and lifestyle modification and optimizing healthcare resources through a multidisciplinary team approach constitute key

\section{Dovepress}

features of the journal. This journal is indexed on American Chemical Society's Chemical Abstracts Service (CAS). The manuscript management system is completely online and includes a very quick and fair peerreview system, which is all easy to use. Visit http://www.dovepress.com/ testimonials.php to read real quotes from published authors.

Submit your manuscript here: http://www.dovepress.com/integrated-blood-pressure-control-journal 